\title{
Decreasing trend in the incidence of serious pneumonias in Finnish children with juvenile idiopathic arthritis
}

\author{
Päivi H. Salonen ${ }^{1,2} \cdot$ Juha H. Salonen ${ }^{3} \cdot$ Hanna Säilä $^{4} \cdot$ Mika Helminen $^{5,6} \cdot$ Miika Linna $^{7}$ - Markku J. Kauppi ${ }^{1,8}$
}

Received: 5 April 2019 / Revised: 25 September 2019 / Accepted: 2 October 2019 / Published online: 16 November 2019

(C) The Author(s) 2019

\begin{abstract}
Objectives Children with juvenile idiopathic arthritis (JIA) may be predisposed to serious pneumonia due to modern diseasemodifying anti-rheumatic treatment. In this nationwide retrospective study with clinical data, we describe the pneumonia episodes among children with JIA.

Methods Patients under 18 years of age with JIA and pneumonia during 1998-2014 were identified in the National Hospital Discharge Register in Finland. Each individual patient record was reviewed, and detailed data on patients with JIA and pneumonia were retrieved, recorded, and analyzed. If the patient was hospitalized or received intravenous antibiotics, the pneumonia was considered serious.

Results There were 157 episodes of pneumonia among 140 children with JIA; 111 episodes (71\%) were serious (80\% in 19982006 and $66 \%$ in 2007-2014). The mean age of the patients was 9 years. Forty-eight percent had active JIA and 46\% had comorbidities. Disease-modifying anti-rheumatic drugs (DMARD) were used at the time of 135 episodes (86\%): methotrexate (MTX) by $62 \%$ and biologic DMARDs (bDMARD) by $30 \%$. There was no significant difference in the use of bDMARDs, MTX and glucocorticoids between the patient groups with serious and non-serious pneumonia episodes. During six of the episodes, intensive care was needed. Two patients $(1.3 \%)$ died, the remaining ones recovered fully.

Conclusions Although the incidence of pneumonia and the use of immunosuppressive treatment among children with JIA increased from 1998 to 2014, the proportion of serious pneumonias in these patients decreased. There was no significant difference in the use of anti-rheumatic medication between patients with serious and non-serious pneumonia.

Key Points

- The incidence of serious pneumonias decreased from 1998 to 2014 among children with juvenile idiopathic arthritis (JIA).

- There was no significant difference in the use of the disease-modifying anti-rheumatic medication between JIA patients with serious and non-serious pneumonias.

- Active JIA, comorbidities, and combination medication were associated with nearly half of the pneumonias.
\end{abstract}

Keywords Biologic agents · Disease-modifying anti-rheumatic drugs $\cdot$ Juvenile idiopathic arthritis $\cdot$ Pneumonia

Salonen P, Säilä H, Salonen J, Helminen M, Linna M (2018) Analysis of 157 pneumonia episodes in Finnish children with juvenile idiopathic arthritis. Scand J Rheumatol 47 (Suppl 129):38

Päivi H. Salonen

paivi.salonen@tuni.fi

1 Faculty of Medicine and Life Science, Tampere University, Tampere, Finland

2 Päijät-Häme Joint Authority for Health and Wellbeing, Terveystie 4, 15870 Lahti, Hollola, Finland

3 Department of Infectious Diseases, Vaasa Central Hospital, Vaasa, Finland
4 Orton Research Institute, Orton Foundation, Helsinki, Finland

5 Research, Development and Innovation Centre, Tampere University Hospital, Tampere, Finland

6 Faculty of Social Sciences, Health Sciences, Tampere University, Tampere, Finland

7 Aalto University, Helsinki, Finland

8 Department of Rheumatology, Päijät-Häme Central Hospital, Lahti, Finland 


\section{Introduction}

The outcome of juvenile idiopathic arthritis (JIA) has improved significantly as medications have developed, much due to the advent of biologic disease-modifying anti-rheumatic drugs (bDMARD) [1]. However, the adverse effects of immunosuppressive medication, including infections, are not fully known. The risk of infections in children with JIA is related to the JIA itself, to concurrent illnesses and to the immunosuppressive treatment [2]. JIA patients without medication have a 2-fold incidence of bacterial infections requiring hospitalization when compared with a control population of similar age [3]. The use of glucocorticoids (GC) increases the risk of serious infections, while the effects of methotrexate (MTX) and the most common group of bDMARDs used to treat pediatric patients, tumor necrosis factor alpha inhibitors (TNFIs), have yielded contradictory results on the occurrence of infections [4]. Modern, effective immunosuppressive medication may increase the risk of serious infections, but it improves, at the same time, the control of JIA and reduces the overall risk of infections [5]. Pneumonia is one of the most common serious infections in children with JIA [5-7].

Recently, we reported that the rate of pneumonia increased in Finnish children with JIA as well as in the general population of corresponding age during the years 1999-2014. At the same time, the use of MTX and bDMARD increased significantly among JIA patients, while the use of GC remained unchanged [8]. For this paper, we have analyzed the patient data from 1998 to 2014 to describe in further detail the severity of pneumonia in this patient group and its relationship, if any, to immunosuppressive medication.

\section{Materials and methods}

\section{Data source}

The data for this national retrospective study was retrieved from the National Hospital Discharge Register (Care Register for Health Care; HILMO) maintained by the National Institute for Health and Welfare. This register has been described in our previous study [8]. Because hospital outpatient data was entered into the HILMO register starting from 1998, our previous register study on the incidence of pneumonia covered the years beginning with 1999 until 2014 to get reliable register information. For the present study, all data available on patients with JIA and radiographically verified pneumonia were retrieved from patient records covering the time from 1998 to 2014.

The coordinating ethics committee of the Helsinki and Uusimaa Hospital District approved the study protocol (228/ 13/03/00/10) and the use of the Care Register for Health Care (HILMO) data.

\section{Study population}

We used the HILMO register to retrieve nationwide data on children younger than 16 years with JIA (diagnose codes M08.0-M08.9) during 1998-2014. They were followed until the day they were 18 years or to the end of 2014 . We linked the identity codes of these patients to the diagnosis of bacterial or undefined pneumonia (J13-J18.9). Pneumonias before the diagnosis of JIA were excluded.

\section{Patient records}

The data on JIA and pneumonia was confirmed by reviewing the individual patient records obtained from the treating hospitals. The study group comprised JIA patients with radiographically verified pneumonia (pulmonary infiltrate) assessed by a radiologist and a pediatrician. We collected the information on JIA and pneumonia from the patient records as described in our previous study [8].

Pneumonia was defined as serious, if the patient was hospitalized or treated with intravenous antibiotics $[9,10]$. The pneumonia was considered hospital-acquired if it developed $48 \mathrm{~h}$ or later after hospital admission for other reasons [11]. The activity of JIA was assessed by scrutiny of the patient record entries of the previous hospital visit or at the time of the pneumonia. JIA was considered inactive if the patient had no active arthritis, no general symptoms (fever, rash, serositis) and no active uveitis, and if the erythrocyte sedimentation rate (ESR) and/or the C-reactive protein (CRP) were within the reference range before the pneumonia diagnosis [12]. Otherwise, the JIA was considered to be active. No activity scores were available. The use of GC was divided into two groups: more than $10 \mathrm{mg}$ or less than $10 \mathrm{mg}$ of prednisolone equivalent per day. Antibiotic therapies started within 2 weeks before pneumonia episodes were included in the analysis. Readmission was defined as a new hospital admission if it occurred within 30 days after hospital discharge for pneumonia [13].

\section{Statistics}

Patient and pneumonia episode characteristics were described by frequencies, percentages, and means (with the respective standard deviations) or medians (with other quartiles). Differences in patient characteristics and anti-rheumatic medication at the time of the pneumonia episodes were analyzed with Fisher's exact test or with the Mann-Whitney test. The risk factors for serious pneumonia were assessed by logistic regression with serious pneumonia as the dependent variable. The independent variables were active JIA, JIA duration (more/less than 2 years), previous recurrent infections, asthma, age (higher/lower than 5 years), combination medication, MTX, bDMARD, GC, previous antibiotic therapy, and 
follow-up period (1998-2006 and 2007-2014). Instability in the regression models due to over-fitting and probable multicollinearity may affect substantially the estimate values and the generalizability of the results. As one solution to this problem, we additionally used logistic regression with a backward selection procedure, where the probability of the Wald statistic was used with the removal $p$ value of 0.10 . Also, the best model based on the Akaike information criterion (AIC) was defined. Analyses were performed with the SPSS statistics software, version 25.0 and with the R-version 3.3.0 (R: A language and environment for statistical computing. R Foundation for Statistical Computing, Vienna, Austria).

\section{Results}

\section{Characteristics of JIA patients with pneumonia}

There were altogether 59,048 JIA patient years during the follow-up. In 1998-2006, the number of children with JIA per year varied between 2292 and 3575 and in 2007-2014 between 3633 and 4511. We identified 157 pneumonia episodes in 140 children with JIA. Of the pneumonia episodes, 111 were serious $(70.7 \%)$ and all except one were communityacquired. Nineteen patients $(13.6 \%)$ had inactive JIA and were not on DMARD treatment. Fourteen JIA patients (10\%) had an episode of pneumonia within the first 3 months of diagnosis. Almost half (45.7\%) of the JIA patients with pneumonia had some concurrent illness; the most common were asthma $(17.9 \%)$ and Down syndrome $(7.1 \%)$ (Table 1). Previous recurrent infections had affected 22 children $(15.7 \%)$.

Altogether 15 JIA patients $(10.7 \%)$ had recurrent pneumonias. They had 32 pneumonia episodes of which 18 (56.3\%) were serious. Comorbidities affected 12 of these patients $(80 \%)$. Two patients had three pneumonias: One of them had aggressive systemic JIA (sJIA) treated with GC (> 10 $\mathrm{mg}$ /day), and one had seronegative polyarthritis and Down syndrome associated with neutropenia and recurrent infections.

\section{JIA medication at the time of pneumonia}

At the time of the pneumonia episodes, $86 \%$ of the patients used DMARDs and 48.4\% combination medication (Table 2). Altogether $68.1 \%$ of bDMARD users took concomitantly MTX and $38.3 \%$ took GC; $8.5 \%$ took a daily dose of $\geq$ $10 \mathrm{mg}$ prednisolone equivalent of GC. The use of MTX increased from 51 to $68 \%$ and of bDMARDs from 15 to $40 \%$ from 1998-2006 to 2007-2014. TNFIs (83\%) were the most common bDMARDs (etanercept 42.6\%). Anti-rheumatic medications with tocilizumab, anakinra, and GC ( $\geq 10 \mathrm{mg} /$ day) were associated exclusively with serious pneumonia episodes (Table 2). Patients stood on DMARDs during 28 out of the $32(87.5 \%)$ recurrent pneumonia episodes: Eight $(25 \%)$ took GC and $13(40.6 \%)$ took bDMARDs. Six patients were treated in the intensive care unit; two of them used bDMARDs and four GCs. Immunosuppressive therapy for JIA (except GC) was always discontinued when pneumonia was treated.

\section{Diagnostic testing}

Altogether 80 blood cultures (BC) were obtained from the patients with serious pneumonia $(72.1 \%)$; of these, five were bacteria-culture positive $(6.3 \%)$, and one was a contamination (1.3\%). Streptococcus pneumoniae was detected in three cases: One patient was in remission without medication; one took a combination of prednisolone (1.25 mg/day), leflunomide, and hydroxychloroquine; and one had active sJIA treated with a combination of prednisolone $(10 \mathrm{mg} /$ day), MTX, and bDMARD. The CRP, ESR, and leucocyte values were within the reference range of one JIA patient on tocilizumab, prednisolone (26 mg/day), and leflunomide despite Staphylococcus aureus sepsis and pneumonia.

A microbiological etiology was verified in 21 episodes (13.4\%): Mycoplasma pneumoniae in six, Influenza A in three, Rhinovirus in two, and Chlamydia pneumoniae in two episodes. Respiratory syncytial and Parainfluenza viruses were detected in one episode each (Table 3). There were no multiresistant microbes. One patient with active JIA treated with prednisolone (10 mg/day), MTX, and certolizumab (third bDMARD) had an episode of complicated pneumonia with prolonged intermittent fever and developed subsequently cerebral Mycobacterium tuberculosis abscesses.

\section{Outcome of pneumonia}

Of the 157 episodes of pneumonia, 105 (66.9\%) were treated with parenteral antibiotics, more than half with cephalosporins (57.1\%). The mean duration of antibiotic treatment was 10 days. Twelve pneumonia episodes $(7.6 \%)$ required two or more consecutive courses of antibiotics.

The median duration of hospital stay was 3 days (range 1114 days). Six episodes were treated in the intensive care unit. Nine pneumonia episodes $(8.1 \%)$ were followed by readmission: Seven were due to pneumonia, one to diarrhea, and one to active JIA. Of the 32 recurrent pneumonia episodes, two led to readmission and three required intensive care. Minimal or moderate pleural effusion was present in ten pneumonia episodes $(6.4 \%)$. None of the patients needed mechanical ventilation, pleural drainage, or other invasive procedures. Four bronchoscopies were performed because of prolonged cough or hemoptysis: Infection was diagnosed in three and primary ciliary dyskinesia (Kartagener syndrome) in one. 
Table 1 Characteristics of 140 individual patients with juvenile idiopathic arthritis and pneumonia at the time of the first pneumonia

\begin{tabular}{llll}
\hline & All patients $(n=140)$ & Serious pneumonia* $(n=101)$ & Non-serious pneumonia* $(n=39)$ \\
\hline Age, mean (SD), years & $9.4(4.8)$ & $9.1(4.9)$ & $10.2(4.5)$ \\
Female, $n(\%)$ & $83(59.3)$ & $59(58.4)$ & $24(61.5)$ \\
JIA duration, median $\left(\mathrm{Q}_{1}-\mathrm{Q}_{3}\right)$, years & $2.6(1.0-6.2)$ & $2.6(1.0-6.9)$ & $2.6(1.0-5.9)$ \\
$<2$ years, $n(\%)$ & $56(40.0)$ & $39(38.6)$ & $17(43.6)$ \\
Systemic arthritis, $n(\%)$ & $10(7.1)$ & $9(8.9)$ & $22(56.4)$ \\
Oligoarthritis, $n(\%)$ & $63(45.0)$ & $41(40.6)$ & $16(41.0)$ \\
Polyarthritis, $n(\%)$ & $64(45.7)$ & $48(47.5)$ & 0 \\
Others & $3(2.1)$ & $3(3.0)$ & $9(23.1)$ \\
Uveitis, $n(\%)$ & $34(24.3)$ & $25(24.8)$ & $16(41.0)$ \\
Active JIA, $n(\%)$ & $67(47.9)$ & $51(50.5)$ & $18(46.2)$ \\
Comorbidities, $n(\%)$ & $64(45.7)$ & $46(45.5)$ & $11(28.2)$ \\
Asthma & $25(17.9)$ & $14(13.9)$ & $3(7.7)$ \\
Down syndrome & $10(7.1)$ & $7(6.9)$ & $1(2.6)$ \\
Iatrogenic adrenal insufficiency & $7(5.0)$ & $6(5.9)$ & $1(2.6)$ \\
Diabetes mellitus & $3(2.1)$ & $2(2.0)$ & 0 \\
IBD & $2(1.4)$ & $2(2.0)$ & $15(14.9)$ \\
Others & $17(12.1)$ & & $2(5.1)$
\end{tabular}

$J I A$ juvenile idiopathic arthritis, $Q_{1}-Q_{3}$ lower-upper quartile, $I B D$ inflammatory bowel disease

*There were no statistically significant differences between serious and non-serious pneumonia groups (Fisher's exact test or the Mann-Whitney test)

Two patients died (1.3\%). In 1998, a 10-year-old girl, who had aggressive seronegative polyarthritis for 9 years, was treated with GC (15 mg/day), azathioprine, and MTX. Since the disease did not remit, she was further treated with an autologous stem cell transplantation after which she required prolonged intensive care period. She developed neutropenia, pneumonia, and Candida albicans sepsis. She died of acute respiratory distress and heart failure. In 2000, a 7-year-old girl

Table 2 Anti-rheumatic medication at the time of 157 pneumonia episodes among patients with juvenile idiopathic arthritis

\begin{tabular}{|c|c|c|c|}
\hline Medication & $\begin{array}{l}\text { All } \\
(n=157)\end{array}$ & $\begin{array}{l}\text { Serious pneumonia episodes } \\
(n=111)\end{array}$ & $\begin{array}{l}\text { Non-serious pneumonia episodes } \\
(n=46)\end{array}$ \\
\hline MTX, $n(\%)$ & $97(61.8)$ & $66(59.5)$ & $31(67.4)$ \\
\hline Duration of MTX use, median $\left(\mathrm{Q}_{1}-\mathrm{Q}_{3}\right)$ (years, $\left.n=66+31\right)$ & $1.6(0.6-3.4)$ & $1.7(0.6-3.3)$ & $1.6(0.5-4.2)$ \\
\hline bDMARD, $n(\%)$ & $47(29.9)$ & $30(27.0)$ & $17(37.0)$ \\
\hline TNFI & $39(24.8)$ & $23(20.7)$ & $16(34.8)$ \\
\hline Tocilizumab & $5(3.2)$ & $5(4.5)$ & 0 \\
\hline Anakinra & $2(1.3)$ & $2(1.8)$ & 0 \\
\hline Abatacept & $1(0.6)$ & 0 & $1(2.2)$ \\
\hline Duration of bDMARD use, median $\left(\mathrm{Q}_{1}-\mathrm{Q}_{3}\right)$ (years, $\left.n=30+17\right)$ & $1.3(0.7-3.3)$ & $1.1(0.5-2.1)^{*}$ & $2.9(0.9-4.6)^{*}$ \\
\hline$\geq 1$ prior biologic agent, $n(\%)$ & $17(10.8)$ & $9(8.1)$ & $8(17.4)$ \\
\hline GC any dose, $n(\%)$ & $26(16.6)$ & $22(19.8)$ & $4(8.7)$ \\
\hline$\geq 10 \mathrm{mg}$ prednisolone equivalent dose of $\mathrm{GC}, n(\%)$ & $8(5.1)$ & $8(7.2)$ & 0 \\
\hline Duration of GC use, median $\left(\mathrm{Q}_{1}-\mathrm{Q}_{3}\right)($ years, $n=22+4)$ & $2.4(0.7-5.1)$ & $2.0(0.6-2.0)$ & $3.6(1.7-4.8)$ \\
\hline Combination medication, $n(\%)$ & $76(48.4)$ & $58(52.3)$ & $18(39.1)$ \\
\hline Without medication, $n(\%)$ & $22(14.0)$ & $17(15.3)$ & $5(10.9)$ \\
\hline
\end{tabular}

$M T X$ methotrexate, $Q_{I^{-}} Q_{3}$ lower-upper quartile, $b D M A R D$ biologic disease-modifying anti-rheumatic drug, $T N F I$ tumor necrosis factor alpha inhibitor, $G C$ glucocorticoid

*The duration of bDMARD use was significantly shorter $(p=0.046)$ in the serious pneumonia group compared to the non-serious group (the MannWhitney test). There were no other statistically significant differences between serious and non-serious pneumonia groups (the Mann-Whitney test or Fisher's exact test). Only those who used anti-rheumatic medication were included in the statistical analysis 
Table 3 Symptoms and laboratory findings at the time of 157 pneumonia episodes among patients with juvenile idiopathic arthritis

\begin{tabular}{|c|c|c|c|c|}
\hline & $\begin{array}{l}\text { All } \\
(n=157)\end{array}$ & $\begin{array}{l}\text { Serious pneumonia } \\
(n=111)\end{array}$ & $\begin{array}{l}\text { Non-serious pneumonia } \\
(n=46)\end{array}$ & $p$ value \\
\hline \multicolumn{5}{|l|}{ Symptom, $n(\%)$} \\
\hline Fever $\left(>38^{\circ}\right)$ & $141(89.8)$ & $104(94.5)^{\mathrm{a}}$ & $37(80.4)$ & 0.014 \\
\hline Cough & $140(89.2)$ & $96(86.5)$ & $44(95.7)$ & 0.155 \\
\hline Dyspnea & $24(15.3)$ & $20(18.0)$ & $4(8.7)$ & 0.222 \\
\hline Chest pain & $16(10.2)$ & $15(13.5)$ & $1(2.2)$ & 0.040 \\
\hline Vomiting & $21(13.4)$ & $19(17.1)$ & $2(4.3)$ & 0.038 \\
\hline Duration of symptoms (days) before diagnosis, median $\left(\mathrm{Q}_{1}-\mathrm{Q}_{3}\right)$ & $6(3-12.3)$ & $5(3-7)$ & $7(4-14)$ & 0.003 \\
\hline Duration of fever (days) before diagnosis median $\left(\mathrm{Q}_{1}-\mathrm{Q}_{3}\right)(n=110+46)$ & $2(1-4)$ & $2(1-4)$ & $2.5(1-4)$ & 0.952 \\
\hline $\mathrm{CRP}$, median $\left(\mathrm{Q}_{1}-\mathrm{Q}_{3}\right), \mathrm{mg} / \mathrm{mL}$ & $77(28-150)$ & $91(41-179)$ & $46(11.3-106.5)$ & $<0.001$ \\
\hline Leucocytes, median $\left(\mathrm{Q}_{1}-\mathrm{Q}_{3}\right)$, E9/L & $11.6(7.0-17.4)$ & $13.1(7.4-18.8)$ & $7.4(6.1-12.2)$ & $<0.001$ \\
\hline Microbiological diagnosis, $n(\%)$ & $21(13.4)$ & $17(15.3)$ & $4(8.7)$ & 0.315 \\
\hline
\end{tabular}

The data was analyzed by Fisher's exact test or by the Mann-Whitney test

$Q_{1}-Q_{3}$ lower-upper quartile, $C R P$ C-reactive protein

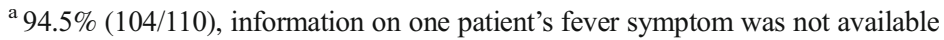

with active sJIA for nearly 5 years was diagnosed with lobar pneumonia and pleuritis. Before the pneumonia, she had been treated with etanercept for 1 year combined with GC and hydroxychloroquine. She died of respiratory failure due to pneumonia.

\section{Comparison of serious and non-serious pneumonia episodes}

During the first half of the follow-up (1998-2006), 49 of 61 $(80 \%)$ and during the latter half (2007-2014) 52 of 79 JIA patients $(66 \%)$ had an episode of serious pneumonia ( $p=$ $0.104)$. There was a trend of children under 5 years being hospitalized more often than older children $(p=0.069)$ (Table 4). There were no significant differences in the other patient characteristics between the serious and non-serious pneumonia groups (Table 1). The children with non-serious pneumonia, in comparison with those with serious pneumonia, had a trend of having previously more often recurrent infections (OR $0.34,95 \%$ CI $0.11-1.04, p=0.058$ ) and asthma (OR 0.39, 95\% CI 0.14-1.11, $p=0.078$ ) (Table 4). The regression model in Table 4 may have been over-fitted. The

Table 4 Comparison of serious and non-serious pneumonias at the time of the first pneumonia in 140 patients with juvenile idiopathic arthritis, logistic regression analysis

\begin{tabular}{|c|c|c|c|c|}
\hline Variable, $n(\%)$ & Serious pneumonia $(n=101)$ & Non-serious pneumonia $(n=39)$ & OR $(95 \% \mathrm{CI})^{*}$ & $p$ value \\
\hline Active JIA & $51(50.5)$ & $16(41.0)$ & $1.44(0.59-3.61)$ & 0.432 \\
\hline JIA duration $<2$ years & $39(38.6)$ & $17(43.6)$ & $0.67(0.23-1.93)$ & 0.458 \\
\hline History of infections & $13(12.9)$ & $9(23.1)$ & $0.34(0.11-1.04)$ & 0.058 \\
\hline Asthma & $14(13.9)$ & $11(28.2)$ & $0.39(0.14-1.11)$ & 0.078 \\
\hline Age $<5$ years & $27(26.7)$ & $6(15.4)$ & $2.93(0.92-9.35)$ & 0.069 \\
\hline Combination medication & $49(48.5)$ & $15(38.5)$ & $3.00(0.83-10.9)$ & 0.095 \\
\hline MTX & $59(58.4)$ & $26(66.7)$ & $0.53(0.24-1.36)$ & 0.183 \\
\hline bDMARD & $27(26.7)$ & $13(33.3)$ & $0.34(0.09-1.22)$ & 0.098 \\
\hline Glucocorticoid & $19(18.8)$ & $4(10.3)$ & $2.69(0.61-12.0)$ & 0.193 \\
\hline Previous antibiotic therapy & $18(17.8)$ & $9(23.1)$ & $0.66(0.24-1.82)$ & 0.424 \\
\hline \multicolumn{5}{|l|}{ Follow-up period } \\
\hline 1998-2006 & $49(80.3)$ & $12(19.7)$ & 1 & \multirow[t]{2}{*}{ \} 0.104} \\
\hline 2007-2014 & $52(65.8)$ & $27(34.2)$ & $0.46(0.18-1.17)$ & \\
\hline
\end{tabular}

Variables remaining in the final model using Akaike information criterion are italicized. Significant variables after backward elimination are in bold JIA juvenile idiopathic arthritis, MTX methotrexate, $B D M A R D$ biologic disease-modifying anti-rheumatic drug, $C I$ confidence interval

*Odds ratios (OR) were assessed by logistic regression with serious pneumonia as the dependent variable 
best result when using the AIC criterion for model selection contained the following variables: follow-up period ( $p=$ $0.030)$, previous recurrent infections $(p=0.032)$, asthma $(p$ $=0.051), \mathrm{GC}(p=0.069)$, and age $(p=0.126)$. When using the backward elimination procedure to the full model, only asthma $(p=0.039)$ and follow-up period $(p=0.047)$ remained in the final model.

The most distinct difference in the use of anti-rheumatic medication before pneumonia episodes was a significantly shorter period of bDMARD use among children with serious pneumonia than non-serious pneumonia $(p=0.046)$ (Table 2). JIA patients with serious pneumonia sought hospital treatment significantly earlier than patients with non-serious pneumonia $(p=0.003)$ (Table 3$)$. The leucocyte $(p<0.001)$ and CRP $(p<0.001)$ values were significantly higher among the children with serious pneumonia than non-serious pneumonia (Table 3). The use of antibiotic therapy before the pneumonia episodes was similar in the two groups (Table 4), and there was no difference with regard to the use of consecutive antibiotic courses.

\section{Discussion}

In this Finnish national register-based, real-world study, there were 157 episodes of mainly community-acquired pneumonia among 140 JIA patients; $71 \%$ of these episodes were serious. In our previous study, the overall incidence of pneumonia increased through 1999-2014, and during the latter half of the follow-up period (2007-2014), it was significantly higher among patients with JIA than in the general population of similar age [8]. Since there were signs of collinearity with some of the target covariates, the model presented in Table 4 was probably over-fitted. The model was reduced with two different methods, and the results corroborate the finding that the rate of pneumonia, defined as serious, in fact decreased from 1998-2006 to 2007-2014 among JIA patients. Maybe treating patterns for pneumonia have shifted towards favoring outpatient care which would decrease the recorded rate of serious pneumonias, since serious pneumonia was defined as pneumonia treated in a hospital or with intravenous antibiotics. On the other hand, maybe shifts in the treatment and follow-up practices of JIA patients have changed in a direction favoring a better contact between patients and the health care system. If so, the health care system would catch upper respiratory infections and incipient pneumonias at an earlier stage, and earlier intervention would reduce the risk of the patient developing serious pneumonia. It is also worth noting that a decrease in pneumonia rates has been reported after introduction of pneumococcal vaccination into the Finnish national vaccination program in 2010 [14].

Active JIA, expressed in terms of the clinical Juvenile Arthritis Disease Activity Score (cJADAS10), is an independent risk factor for serious infections [9]. Within 2 years after
JIA has been diagnosed and treated, on average, $78 \%$ of patients experience inactivation of the disease; for patients with rheumatoid-factor positive polyarthritis, the figure is $48 \%$ [15]. In this study, nearly half of the JIA patients had active disease (Table 1) and 10\% of the pneumonia episodes occurred within 3 months after JIA had been diagnosed. A study from Germany concluded that patients on immunosuppressive therapy have a high risk for pneumonia and that patients with $\geq 2$ at-risk conditions (e.g., rheumatoid arthritis, asthma, Down syndrome, diabetes) have a similar or higher risk of pneumonia compared with patients on immunosuppressive therapy [16]. Our results corroborate these findings: $46 \%$ of our patients had some concurrent illness or morbidity, and $16 \%$ had had recurrent infections. Of the recurrent pneumonias, $80 \%$ occurred in patients with some comorbidity and $88 \%$ in patients on DMARD therapy.

Children hospitalized for community-acquired pneumonia (CAP) have frequent asthma [17]. Hospitalization for CAP is greatest in children under 5 years of age and one third of them have asthma $[18,19]$. In our study, there was a trend for more frequent hospitalization of children under 5 years than older children. Altogether 18\% of JIA patients with pneumonia had asthma and of them 56\% were hospitalized (Table 1). There was a trend for asthma being more common in the non-serious pneumonia group (OR 0.39, 95\% CI 0.14-1.11, $p=0.078$ ) (Table 4), which could be due to more intensive treatment of asthma and pneumonia.

Altogether 7\% of JIA patients with pneumonia had Down syndrome. The prevalence of Down syndrome in Finland is $0.27 \%$ [20], and the prevalence of JIA among children with Down syndrome is 6 -fold $(0.87 \%)$ compared with JIA in the general population [21]. Thus, we corroborated previous findings [16] that JIA patients with Down syndrome have a higher than average risk of pneumonia.

In our study, there was a trend for a history of infections being associated more often with non-serious than serious pneumonia (OR 0.34, 95\% CI 0.11-1.04, $p=$ 0.058 ) in JIA patients. Antibiotic therapy may be started in these children earlier and more efficiently than before. The rate of outpatient antibiotic prescriptions for children on MTX and/or TNFI is about two times higher than the rate among children not on this medication [22]. We found no significant difference in the previous use of antimicrobials between the serious and non-serious pneumonia groups (Table 4).

Effective treatment of JIA has increased during the last 20 years, but still most pediatric JIA patients have active disease and need long-term treatment [23-25]. In Finland, $72 \%$ of recently diagnosed JIA patients used MTX about a decade ago [26], and $66 \%$ of adolescents and young adults with JIA took MTX, 29\% bDMARDs, and 9\% GCs [23]. In our study, combination medication was used by nearly half of the patients with JIA and pneumonia and, in the latter half of the follow-up, $40 \%$ of JIA patients were on bDMARDs. Serious 
infections account for one third of all serious adverse events attributed to bDMARDs [27]. In a recent study, three out of 307 children with JIA on bDMARD therapy $(1 \%)$ had a serious infection during 1 year of follow-up [28].

When immunosuppressive drugs are combined, the total immunosuppressive effect is at least as powerful as with the most immunosuppressive agent used alone [5]. A study on US Medicaid data reported that there was no significant risk for infections requiring hospital treatment among patients on TNFI monotherapy or on TNFI in combination with MTX [7]. Nor did we find any significant difference in the occurrence of serious and non-serious pneumonia episodes in relation to the use of MTX and bDMARDs. In adults with rheumatoid arthritis, a dose-related association between GC use and the risk of hospitalization for pneumonia has been reported [29]. In the present study, daily doses of GC $\geq 10 \mathrm{mg}$ were in use only at the time of the serious pneumonia episodes (Table 2), which would support this observation.

Serious infections in adults with rheumatic diseases usually occur during the first 6 months after start of TNFI therapy [30]. A recent study pointed out that when TNFIs are introduced into the treatment of JIA in children, the risk of serious infections rises 2.7-fold compared with the introduction of DMARDs; here, the mean follow-up time was 307 days [4]. In our study, the mean duration of bDMARD use was significantly shorter $(p=0.046)$ among children with serious pneumonia episodes than nonserious pneumonia episodes (Table 2).

The microbiological diagnosis of pneumonia is challenging. The current management guidelines for CAP recommend $\mathrm{BCs}$ if the patient is hospitalized and the pneumonia is moderate or severe [31]. In a multicenter study, BCs were taken of $56 \%$ of the children hospitalized for CAP, and bacteremia was present in 7\% [32]. In our study, BCs were taken of $72 \%$ of the patients with five positive findings in children with serious pneumonia (6\%). CRP was obtained of nearly all JIA patients with pneumonia (Table 3). CRP and the leucocyte values do not necessarily predict the degree of severity of the lung infection in patients taking bDMARDs [10]. In agreement with this, there was one patient on tocilizumab who had Staphylococcus aureus sepsis, but whose CRP and leucocyte values were within the reference range.

In our study, there were no suppurative complications and none of the patients needed pleural drainage. The JIA patients recovered largely well on antibiotics, although prolonged courses of antibiotic therapy were needed in 12 episodes (7.6\%) and hospital readmission in nine (8\%). Up to $8 \%$ of children with pneumonia are readmitted for hospital treatment, and the rate of readmission increases if the patient has chronic comorbidities [13]. Abinun et al. [33] described four patients with refractory JIA on combined DMARD therapy who developed unexpectedly a severe infection and died. In our series, two patients died in the beginning of the follow-up; both had disabling JIA and required long-term multiple and combined DMARD therapy, including a dose of $\mathrm{GC} \geq 10 \mathrm{mg} /$ day.

To our knowledge, this is the first detailed, nationwide, real-world study on JIA and pneumonia. The strength of our study is that it is based on comprehensive national register data which covers the entire hospital network in Finland. Pediatric rheumatologists attend to children with JIA in the hospitals, and the appropriate ICD diagnoses are available from the HILMO register. In addition to register data, we collected detailed data from patient records and analyzed only radiographically verified pneumonias.

On the other hand, register data may include errors of diagnosis, although we checked painstakingly all registry data from individual patient records. The clinical data on patient records was also incomplete, e.g., data on growth, vaccinations, and clinical signs of pneumonia were only partially available. Therefore, it was not possible to define the severity of pneumonia based on clinical signs, and general guidelines (e.g., American or British Thoracic Society guidelines) could not be used. Because an etiological diagnosis of pneumonia is often difficult to obtain and because this study was retrospective, we focused on bacterial pneumonia ICD codes. Unfortunately, it was not feasible to compare JIA patients with and without pneumonia because selecting appropriate controls would have been an unsurmountable task. Milder cases of pneumonia are treated by open-care physicians, and thus, this data is not available. Overall, the rather low number of pneumonia episodes did not allow for all statistical calculations that would have been of interest, like analyzing the subtypes of JA.

\section{Conclusion}

Despite the increasing incidence of pneumonia and use of immunosuppressive treatment among children with JIA, the proportion of serious pneumonias in these patients has decreased over time. Active JIA, comorbidities and combination medication were associated with nearly half of the pneumonias. There was no significant difference in the use of bDMARD, MTX, or GC between patients with serious and non-serious pneumonia, but this observation needs to be corroborated by further studies. Clinicians should always keep in mind the possibility of serious infectious complications in these immunocompromised patients.

Acknowledgments This study was supported by grants from the Finnish Cultural Foundation and the Finnish Society for Rheumatology.

\section{Compliance with ethical standards}

The coordinating ethics committee of Helsinki and Uusimaa Hospital District approved the study protocol (228/13/03/00/10) and the use of the Care Register for Health Care (HILMO) data.

Disclosures None. 
Open Access This article is distributed under the terms of the Creative Commons Attribution 4.0 International License (http:// creativecommons.org/licenses/by/4.0/), which permits unrestricted use, distribution, and reproduction in any medium, provided you give appropriate credit to the original author(s) and the source, provide a link to the Creative Commons license, and indicate if changes were made.

\section{References}

1. Ravelli A, Martini A (2007) Juvenile idiopathic arthritis. Lancet 369:767-778

2. Horneff G (2015) Biologic-associated infections in pediatric rheumatology. Curr Rheumatol Rep 17:66

3. Beukelman T, Xie F, Chen L et al (2012) Rates of hospitalized bacterial infection associated with juvenile idiopathic arthritis and its treatment. Arthritis Rheum 64:2773-2780

4. Lee W, Lee TA, Suda KJ, Calip GS, Briars L, Schumock GT (2018) Risk of serious bacterial infection associated with tumour necrosis factor-alpha inhibitors in children with juvenile idiopathic arthritis. Rheumatology (Oxford) 57:273-282

5. Davies HD (2016) Infectious complications with the use of biologic response modifiers in infants and children. Pediatrics 138

6. Davies R, Southwood TR, Kearsley-Fleet L, Lunt M, Hyrich KL (2015) Medically significant infections are increased in patients with juvenile idiopathic arthritis treated with etanercept: results from the British Society for Paediatric and Adolescent Rheumatology Etanercept Cohort Study. Arthritis Rheum 67:2487-2494

7. Beukelman T, Xie F, Baddley JW et al (2016) The risk of hospitalized infection following initiation of biologic agents versus methotrexate in the treatment of juvenile idiopathic arthritis. Arthritis Res Ther 18:210

8. Salonen PH, Säilä H, Salonen JH, Linna M, Helminen M, Kauppi MJ (2018) Pneumonia in children with juvenile idiopathic arthritis in Finland 1999-2014: a nationwide retrospective register linkage study. Clin Exp Rheumatol 36:502-507

9. Becker I, Horneff G (2017) Risk of serious infection in juvenile idiopathic arthritis patients associated with tumor necrosis factor inhibitors and disease activity in the German Biologics in Pediatric Rheumatology Registry. Arthritis Care Res 69:552-560

10. Woerner A, Ritz N (2013) Infections in children treated with biological agents. Pediatr Infect Dis J 32:284-288

11. Scott JAG, Wonodi C, Moïsi JC et al (2012) The definition of pneumonia, the assessment of severity, and clinical standardization in the Pneumonia Etiology Research for Child Health Study. Clin Infect Dis 54:S10-S116

12. Wallace CA, Giannini EH, Huang B, Itert L, Ruperto N (2011) American College of Rheumatology provisional criteria for defining clinical inactive disease in select categories of juvenile idiopathic arthritis. Arthritis Care Res 63:929-936

13. Neuman MI, Hall M, Gay JC et al (2014) Readmissions among children previously hospitalized with pneumonia. Pediatrics 134: 100-109

14. Palmu AA, Rinta-Kokko H, Nohynek H, Nuorti JP, Kilpi TM, Jokinen J (2017) Impact of ten-valent pneumococcal conjugate vaccine on pneumonia in Finnish children in a nation-wide population-based study. PLoS ONE 12:e172690

15. Guzman J, Oen K, Tucker LB et al (2015) The outcomes of juvenile idiopathic arthritis in children managed with contemporary treatments: results from the ReACCh-Out cohort. Ann Rheum Dis 74: $1854-1860$

16. Pelton SI, Shea KM, Farkouh RA et al (2015) Rates of pneumonia among children and adults with chronic medical conditions in Germany. BMC Infect Dis 15:470
17. Wilson KM, Torok MR, Localio R et al (2015) Hospitalization for community-acquired pneumonia in children: effect of an asthma codiagnosis. Hosp Pediatr 5:415-422

18. Leyenaar JK, Lagu T, Shieh M, Pekow PS, Lindenauer PK (2014) Management and outcomes of pneumonia among children with complex chronic conditions. Pediatr Infect Dis J 33:907-911

19. Jain S, Williams DJ, Arnold SR et al (2015) Community-acquired pneumonia requiring hospitalization among U.S. children. N Engl J Med 372:835-845

20. The National Institute of Health and Welfare (2018) The Finnish Register of Congenital Malformations 2014. http://www.thl.fi/ statistics/congenitalmalformations

21. Juj H, Emery H (2009) The arthropathy of Down syndrome: an underdiagnosed and under-recognized condition. J Pediatr 154: 234-238

22. Ringold S, Grant S, Girdish C, Wallace CA, Sullivan SD (2013) Injectable tumor necrosis factor $\alpha$ inhibitors and outpatient antimicrobial use in children with rheumatic diseases: analyses of prescription claims from a pharmacy benefit manager database. Arthritis Care Res 65:1880-1884

23. Vidqvist K, Malin M, Varjolahti-Lehtinen T, Korpela MM (2013) Disease activity of idiopathic juvenile arthritis continues through adolescence despite the use of biologic therapies. Rheumatology (Oxford) 52:1999-2003

24. Mannion ML, Xie F, Curtis JR, Beukelman T (2014) Recent trends in medication usage for the treatment of juvenile idiopathic arthritis and the influence of tumor necrosis factor inhibitors. J Rheumatol 41:2078-2084

25. Nordal E, Zak M, Aalto K et al (2011) Ongoing disease activity and changing categories in a long-term nordic cohort study of juvenile idiopathic arthritis. Arthritis Rheum 63:2809-2818

26. Pohjankoski H, Latva K, Kautiainen H et al (2011) First-year purchases of disease-modifying drugs of incident patients with chronic juvenile arthritis in Finland. Clin Exp Rheumatol 29:878-881

27. Tarkiainen $M$, Tynjälä $P$, Vähäsalo $P$, Lahdenne $P$ (2015) Occurrence of adverse events in patients with JIA receiving biologic agents: long-term follow-up in a real-life setting. Rheumatology (Oxford) 54:1170-1176

28. Aygun D, Sahin S, Adrovic A et al (2019) The frequency of infections in patients with juvenile idiopathic arthritis on biologic agents: 1-year prospective study. Clin Rheumatol 38:1025-1030

29. Wolfe F, Caplan L, Michaud K (2006) Treatment for rheumatoid arthritis and the risk of hospitalization for pneumonia: Associations with prednisone, disease-modifying antirheumatic drugs, and antitumor necrosis factor therapy. Arthritis Rheum 54:628-634

30. Galloway JB, Hyrich KL, Mercer LK et al (2011) Anti-TNF therapy is associated with an increased risk of serious infections in patients with rheumatoid arthritis especially in the first 6 months of treatment: updated results from the British Society for Rheumatology Biologics Register with special emphasis on risks in the elderly. Rheumatology (Oxford) 50:124-131

31. Bradley JS, Byington CL, Shah SS et al (2011) The management of community-acquired pneumonia in infants and children older than 3 months of age: clinical practice guidelines by the Pediatric Infectious Diseases Society and the Infectious Diseases Society of America. Clin Infect Dis 53:25

32. Myers AL, Hall M, Williams DJ et al (2013) Prevalence of bacteremia in hospitalized pediatric patients with community-acquired pneumonia. Pediatr Infect Dis J 32:736-740

33. Abinun M, Lane JP, Wood M, Friswell M, Flood TJ, Foster HE (2016) Infection-related death among persons with refractory juvenile idiopathic arthritis. Emerg Infect Dis 22:1720-1727

Publisher's note Springer Nature remains neutral with regard to jurisdictional claims in published maps and institutional affiliations. 